Bull. Mater. Sci., Vol. 7, No. 2, July 1985, pp. 95-100. (C) Printed in India.

\title{
A minimal network model for creep
}

\author{
M KRISHNAMOORTHY and V BALAKRISHNAN ${ }^{+*}$ \\ Department of Physics, Madura College, Madurai 625011, India \\ ${ }^{\dagger}$ Department of Physics, Indian Institute of Technology, Madras 600036, India.
}

MS received 2 March 1985

\begin{abstract}
We present a minimal rheological network model for the creep function in plastic flow. The model has a very small number of elements-a maximum of two linear springs, two Newtonian dashpots and a frictional mass. No non-Newtonian dashpots or other nonlinear elements are invoked. A wide variety of creep-curve shapes including those with inflection points across which the curvature changes sign are shown to be reproduced by the model and its sub-cases.
\end{abstract}

Keywords. Rheology; mechanical networks; creep curves.

\section{Introduction}

Mechanical networks provide a very convenient phenomenological approach to the modelling of various kinds of rheological behaviour in solids. The most commonly used network elements are the Hookean spring, the Newtonian dashpot and the St. Venant body, which is a mass that moves under an external force against a resisting frictional force (Reiner 1969). All these elements are linear: the stress $\sigma$ and the corresponding strain $\varepsilon$ bear linear functional relationships to each other (the St. Venant body has a threshold stress below which its response vanishes, owing to the friction). Networks comprising only springs and dashpots suffice to model the behaviour of all anelastic and linear viscoelastic materials with discrete strain or stress relaxation spectra (Gross 1968; Lockett 1972; Vinogradov and Malkin 1980). For more complicated kinds of deformation behaviour (such as plasticity) it is necessary to introduce also the third basic element in the networks. In addition, one may have to consider networks with nonlinear springs, non-Newtonian dashpots, as well as other elements that are less frequently invoked, if it is desired to extend this empirical approach to cover the diversity of rheological behaviour that occurs in practice. For instance, a host of problems in deformation kinetics may be handled with the help of non-Newtonian dashpots with suitable constitutive relationships between $\sigma$ and $\varepsilon$ (Krausz and Eyring 1975).

As in the case of lumped-parameter electrical circuits, a given mechanical response function can be simulated in principle by an infinite number of networks. The equivalent-network approach to modelling is therefore useful in practice only if one minimizes the redundancy and maximizes the simplicity in each case. This task is not always a straightforward one, and so it is frequently not given the attention it deserves,

\footnotetext{
* To whom all correspondence should be addressed.
} 
as one may gather from a perusal of the rheological literature. It would therefore be useful to do the following: to find the 'minimal' equivalent networks corresponding to various generic forms of mechanical response, so that one may characterize the materials exhibiting such behaviour by an optimal set of effective lumped parameters. Once these standard models are established, it becomes meaningful to compare numerical values of the relevant parameters for different materials in the same class of deformation behaviour. Such standard models are well known for anelastic and linear viscoelastic materials: the Kelvin (or Voigt) element, the Maxwell element, the 'standard linear solid', the Burgers model, etc. What we propose here are similar simple but universal models for the kinds of creep behaviour encountered most frequently in practice.

\section{A minimal network model for creep}

\subsection{The creep curve}

In a creep experiment, the strain response to an applied stress $\sigma(t)=\sigma_{0} \theta(t)$ is measured as a function of time $[\theta(t)$ is the unit step function]. It is well known (see, e.g., Gittus 1975) that most metals exhibit a creep curve that is typically of the form shown in figure 1. After a possible 'instantaneous' response, there is the primary creep region, associated with multiplication in the number density of a relevant defect species (such as dislocations). This is followed by a region of secondary creep (owing to easy glide, for instance), and finally a steeply rising tertiary creep prior to failure. While the physical mechanisms responsible for these regimes are numerous, and differ in detail from one material to another, the form of the curve is quite universal. It is to be noted that the curvature of the creep function changes sign. This is typical of plastic deformation. We have proved elsewhere (Krishnamoorthy and Balakrishnan 1983) that anelastic and linear viscoelastic materials cannot exhibit such behaviour. Networks corresponding to these materials must have response functions whose curvature does not change sign. In

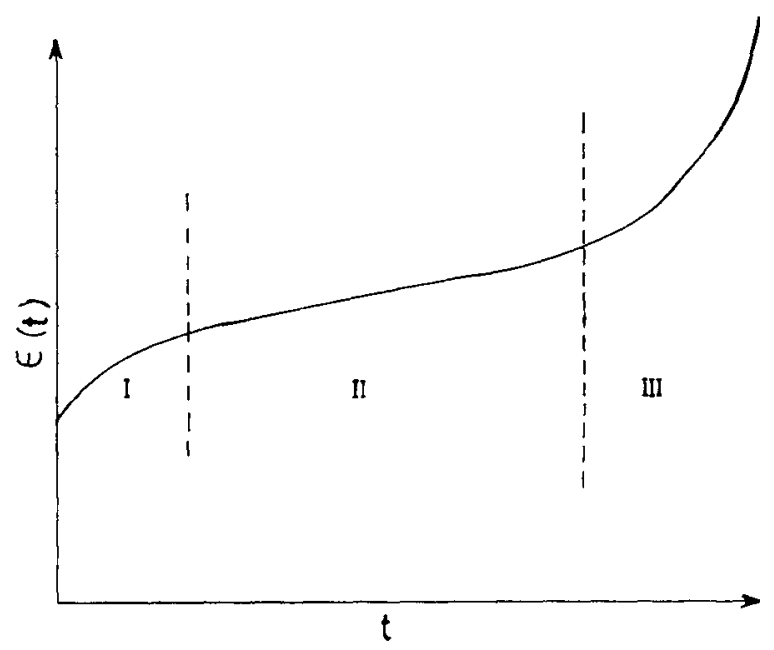

Figure 1. Typical form of the creep behaviour of a metal, with the regions demarcated. 
particular, the creep function is always concave downwards (or else is linear, as in the extreme case of a Maxwell element). The occurrence of one or more points of inflection on the creep curve is thus a significant characteristic of plastic deformation that must be reproduced in phenomenological modelling.

\subsection{Notation}

We shall denote a Hookean spring by $H$, a Newtonian dashpot by $N$, and a St. Venant body by $S$. The basic anelastic element is the Kelvin or Voigt model, consisting of a spring and dashpot in parallel: symbolically, $K=H \mid N$. The visco-elastic counterpart to this is the Maxwell element (spring and dashpot in series), $M=H-N$. The strain responses of $H, N$ and $S$ to an applied stress are given, respectively, by $\varepsilon=\sigma / E, \dot{\varepsilon}=\sigma / \eta$ and $\ddot{\varepsilon}=\left(\sigma-\sigma_{k}\right) \theta\left(\sigma-\sigma_{s}\right) / \mu$, where $E, \eta$ and $\mu$ are constants. $\sigma_{s}$ and $\sigma_{k}\left(\right.$ with $\left.\sigma_{s}>\sigma_{k}\right)$ are the threshold stresses representing the static and dynamic (kinetic) frictional forces on $S$. In what follows, it is to be understood that the steady applied stress $\sigma_{0}$ is greater than $\sigma_{s}$.

\subsection{The model}

After some experimentation, we have found that the simplest (and yet very comprehensive) model that exhibits a creep curve of the shape shown in figure 1 is just $(S \mid M)-K$. The addition of an extra spring in series will reproduce the possible instantaneous strain response as well, but this is a trivial feature as far as network modelling is concerned, and will therefore be omitted. The model is sketched and the network parameters are labelled in figure 2 . On solving the coupled differential equations pertaining to the model, we obtain the following solution for the strain as a function of time. Let

$$
u=\left[\left(E_{1}^{2} / 4 \eta_{1}^{2}\right)-\left(E_{1} / \mu\right)\right]^{1 / 2} .
$$

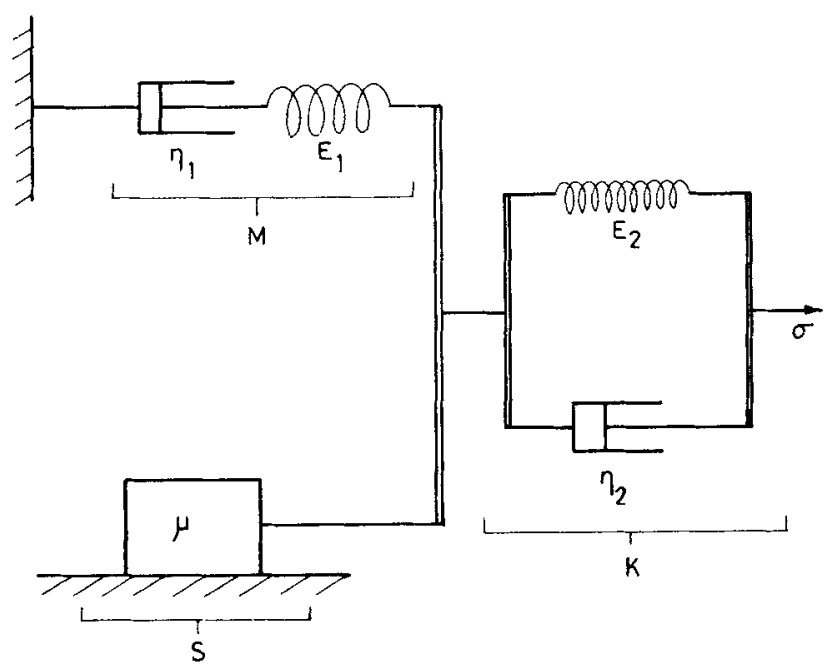

Figure 2. The basic network $(S \mid M)-K$. The parameter $\mu$ depends on the mass and the physical dimensions of the St. Venant body. 
Then

$$
\varepsilon(t)=\varepsilon_{1}(t)+\varepsilon_{2}(t)
$$

where

$$
\begin{aligned}
\varepsilon_{1}(t) /\left(\sigma_{0}-\sigma_{k}\right)= & \frac{t}{\eta_{1}}+\frac{1}{E_{1}}-\frac{\mu}{\eta_{1}^{2}}+\exp \left(-\frac{E_{1} t}{2 \eta_{1}}\right)\left[\frac{1}{E_{1}}\left(\frac{\mu E_{1}}{\eta_{1}^{2}}-1\right)\right. \\
& \left.\cosh u t+\frac{1}{2 u \eta_{1}}\left(\frac{\mu E_{1}}{\eta_{1}^{2}}-3\right) \sinh u t\right]
\end{aligned}
$$

and

$$
\varepsilon_{2}(t) / \sigma_{0}=\left(1 / E_{2}\right)\left[1-\exp \left(-E_{2} t / \eta_{2}\right)\right]
$$

As we shall see, a remarkable variety of creep function forms is encompassed by this single model and its special cases. The analysis of the solution is most transparent if carried out in terms of these special cases, which are also of interest in their own right.

\section{Special cases}

\subsection{The model $S \mid M$ and its sub-cases}

To understand how the inflection point arises in the creep curve, let us omit the Kelvin element for the moment, and consider the network $S \mid M$. The creep function is then $\varepsilon_{1}(t) /\left(\sigma_{0}-\sigma_{k}\right)$. In the parameter regime $u^{2} \geqslant 0$ or $\mu E_{1} \geqslant 4 \eta_{1}^{2}$, the creep function is always concave upwards. The interesting regime is $u^{2}<0$, in which this is no longer true. There is an inflection point at time $t=v^{-1}\left(\pi-\tan ^{-1}\left(2 \eta_{1} v / E_{1}\right)\right)$ where $u=i v$. The solution for $\varepsilon(t)$ remains formally the (analytic) continuation of (3) to $u=i v$, but only in a finite range of $t$. It is clear that the St. Venant body cannot move in a direction opposite to that of the applied stress. The incorporation of this physical consideration is the simplest way of deducing the creep function correctly at larger values of $t$. The point is illustrated by removing even the dashpot in $M$, so that we are left with $S \mid H$. This corresponds to the limit $\eta_{1} \rightarrow \infty$ in (3). (We have already specified that $u^{2}=-v^{2}<0$ ). The creep function is then

$$
\varepsilon(t) /\left(\sigma_{0}-\sigma_{k}\right)=\left(1 / E_{1}\right)\left[1-\cos \left(E_{1} / \mu\right)^{1 / 2} t\right]
$$

in the range $0 \leqslant t \leqslant \pi\left(\mu / E_{1}\right)^{1 / 2}$, beyond which it saturates to the value $2 / E_{1}$, as shown in figure $3(\mathrm{a})$.

We can recover also a monotonically increasing creep function that has the viscoelastic asymptotic behaviour $\sim t$, by removing $H$ instead of $N$ to obtain $S \mid N$. This is achieved by taking the limit $E_{1} \rightarrow \infty$ in (3). We find on simplification

$$
\varepsilon(t) /\left(\sigma_{0}-\sigma_{k}\right)=\left(\eta_{1} / \mu^{2}\right)\left[\left(\eta_{1} t / \mu\right)-1+\exp \left(-\eta_{1} t / \mu\right)\right],
$$

which is sketched in figure $3(\mathrm{~b})$. As $\boldsymbol{u}^{2} \rightarrow+\infty$ in this case, the creep function is concave upwards throughout. It begins with a quadratic dependence on $t$ and approaches a linear one asymptotically. We have discussed elsewhere (Balakrishnan 1979) the reasons why a functional form like (6) may be regarded as the fundamental one for linear visco-elasticity, and also indicated the physical circumstances under which plastic flow can exhibit a similar kind of time dependence. 


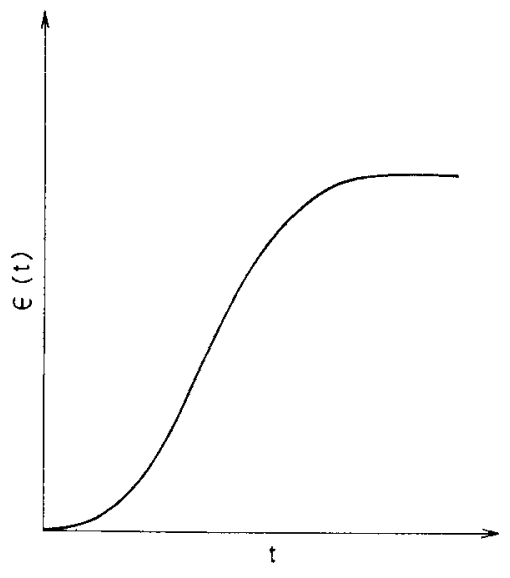

(o)

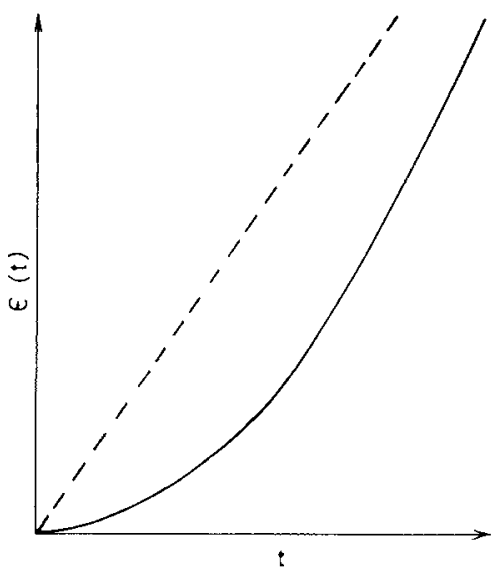

(b)

Figure 3. Creep function for (a) the network $S \mid H$; (b) the network $S \mid N$.

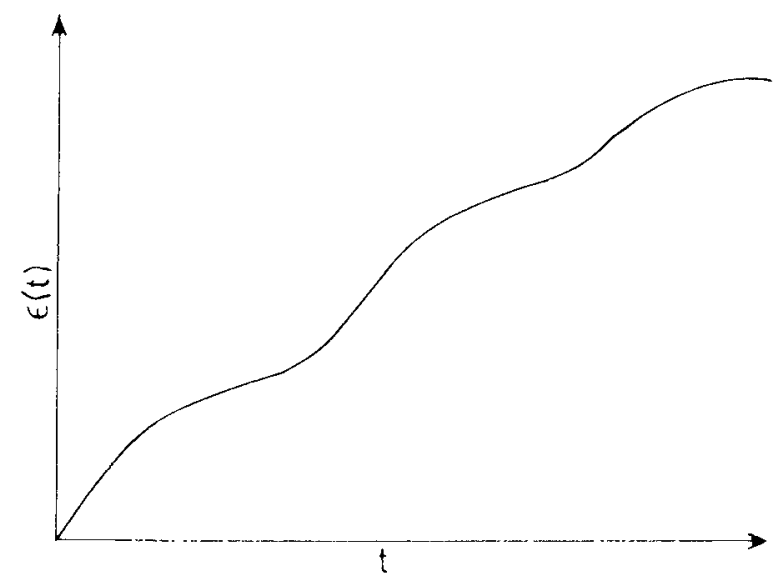

Figure 4. Creep function for polycrystalline lead (Andrade and Margrave 1968), modelled by the network $(S \mid H)-K$.

\subsection{The model ( $S \mid H)-K$ and the creep of polycrystalline lead}

Polycrystalline metals sometimes exhibit a creep curve with more than one inflection point. A typical case is that of polycrystalline lead (Andrade and Margrave 1968), for which a schematic creep curve is shown in figure 4 . This behaviour is simulated by a special case of our basic model in which the dashpot of the Maxwell element is absent. The model is thus represented by $(S \mid H)-K$, and is attained by letting $\eta_{1} \rightarrow \infty$ in the solution (2)-(4). The resulting creep function is directly written down from (4) and (5) (the comment immediately following (5) again applies). What is interesting is that upto two inflection points are possible in the creep curve, depending on the value of the parameter $\mu E_{2} / \eta_{2}^{2}$. 


\subsection{Variants of the model}

Just as the basic network for anelasticity (the standard linear solid) can itself be cast in more than one configuration, so can our minimal model of figure 2, without increasing the number of network parameters. We may thus proceed to compute the strain responses of combinations like $(S \mid K)-M,(S-K) \mid M$, etc. and their sub-cases, in order to find such equivalent configurations. However, as these must lead to the same mechanical response functions, any one of them suffices for the purposes of phenomenological modelling.

\section{Acknowledgements}

This work was commenced and partially carried out at the Reactor Research Centre, Kalpakkam. We thank Dr G Venkataraman for his kind interest and encouragement.

\section{References}

Andrade E N, Da C and Margrave M 1968 Proc. R. Soc. A304 1

Balakrishnan V 1979 Pramana 13545

Gittus J 1975 Creep, viscoelasticity and creep fracture in solids (London: Applied Science)

Gross B 1968 Mathematical structure of the theories of viscoelasticity (Paris: Hermann)

Krausz A S and Eyring H 1975 Deformation kinetics (New York: Academic Press)

Krishnamoorthy M and Balakrishnan V 1983 Philos. Mag. B48 L11

Lockett F J 1972 Nonlinear viscoelastic solids (London: Academic Press)

Reiner M 1969 Deformation, strain and flow (London: Lewis)

Vinogradov G V and Malkin A Ya 1980 Rheology of polymers (Moscow: Mir) 